\title{
FDTD Method for Obliquely Incident Electromagnetic Wave in Time-Varied Plasma
}

Liang Zhao

College of Aerospace Science and Engineering, National University of Defense Technology, Changsha 410073, China imu-zhl@163.com

\section{Xiu-Xiang Liu}

Science and Technology on Space Physics Laboratory, Beijing 100076, China

\author{
Han-Sheng Su \\ Science and Technology on Space Physics Laboratory, \\ Beijing 100076, China
}

in plasma under different incident angle is analyzed.

\section{ONE-DimenSIONAL METHOD FOR OBLIQUELY INCIDENT EM WAVE IN TIME-VARIED PLASMA}

The diagram of obliquely incident EM wave in plasma is shown in Fig. 1, medium 1 and medium $n+1$ in the figure is vacuum, and medium $2 \sim \mathrm{n}$ are layered plasma. The incident angle of EM wave is $\theta$, and $\mathrm{k}$ is wave number.

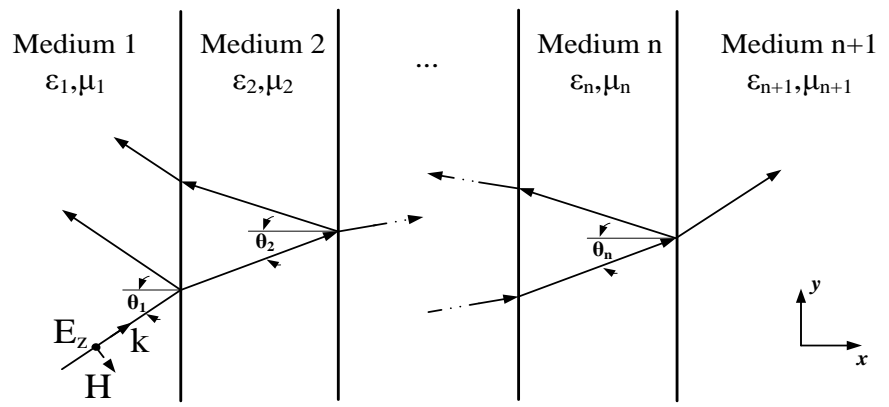

Figure 1. Diagram of obliquely incident TE wave in plasma.

Two-dimensional Maxwell equation for unmagnified TE wave in plasma is

$$
\begin{aligned}
& \frac{\partial E_{z}}{\partial y}=-\mu_{0} \frac{\partial H_{x}}{\partial t} \\
& \frac{\partial E_{z}}{\partial x}=\mu_{0} \frac{\partial H_{y}}{\partial t} \\
& \frac{\partial H_{y}}{\partial x}-\frac{\partial H_{x}}{\partial y}=\varepsilon_{0} \frac{\partial E_{z}}{\partial t}+J_{z} \\
& \frac{\partial J_{z}}{\partial t}+v J_{z}=\varepsilon_{0} \omega_{p}^{2} E_{z}
\end{aligned}
$$


$J_{z}$ Is current density. Take Fourier transform for $3^{\text {rd }}$ and $4^{\text {th }}$ equations in Eq. 1

$$
\begin{gathered}
\frac{\partial \boldsymbol{H}_{y}}{\partial x}-\frac{\partial \boldsymbol{H}_{x}}{\partial y}=j \omega \varepsilon_{0} \boldsymbol{E}_{z}+\boldsymbol{J}_{z} \\
\boldsymbol{J}_{z}=\varepsilon_{0} \frac{\omega_{p}^{2}}{j \omega+v} \boldsymbol{E}_{z}
\end{gathered}
$$

Take Eq. 3 into Eq. 2

$$
\frac{\partial \boldsymbol{H}_{y}}{\partial x}-\frac{\partial \boldsymbol{H}_{x}}{\partial y}=j \omega \varepsilon_{0}\left(1+\frac{\sigma_{n}(\omega)}{j \omega \varepsilon_{0}}\right) \boldsymbol{E}_{z}=j \omega \varepsilon_{n} \boldsymbol{E}_{z}
$$

$\varepsilon_{n}$ Is relative permittivity of the $\mathrm{n}^{\text {th }}$ layer plasma, $\sigma(\omega)=\varepsilon_{0} \frac{\omega_{p}^{2}}{j \omega+v}$. Take differential and Fourier transform for $1^{\text {st }}$ equation in Eq. 1,

$$
\frac{\partial^{2} \boldsymbol{E}_{z}}{\partial y^{2}}=-k_{n y}^{2} \boldsymbol{E}_{z}=-j \omega \mu_{0} \frac{\partial \boldsymbol{H}_{x}}{\partial y}
$$

From phase match in layered medium theory, the tangential value of wave vector in different layer is equal, so $k_{n y}=k_{1 y}=k_{1} \sin \theta$, take Eq. 4 into Eq. 5 and introduce incident angle,

$$
\frac{\partial \boldsymbol{H}_{y}}{\partial x}=j \omega \varepsilon_{n} \boldsymbol{E}_{z}-j \omega \varepsilon_{0} \varepsilon_{1} \sin ^{2} \theta \boldsymbol{E}_{z}
$$

Take Fourier transform for Eq. 6

$$
\left.\begin{array}{l}
\frac{\partial E_{z}}{\partial x}=\mu_{0} \frac{\partial H_{y}}{\partial t} \\
\frac{\partial H_{y}}{\partial x}=\left(c f \cdot \varepsilon_{0}\right) \frac{\partial E_{z}}{\partial t}+J_{z} \\
\frac{\partial J_{z}}{\partial t}+\nu J_{z}=\varepsilon_{0} \omega_{p}^{2} E_{z}
\end{array}\right\}
$$

In Eq. 11

Take Fourier (4) 4 nansform for $3^{\text {rd }}$ equation in Eq. 10, and then take inverse Fourier transform under the assumption of $\ddot{E}(t)$ is linear

$$
\begin{aligned}
J_{z}^{n+1}+J_{z}^{n} & =\left(\sigma^{0}-\xi^{0}\right) \ddot{E}_{z}^{n+1}+\xi^{0} \ddot{E}_{z}^{n}+\sum_{m=0}^{n-1}\left[\ddot{E}_{z}^{n-m}\left(\sigma^{m}+\sigma^{m+1}\right)\right. \\
& \left.+\left(\ddot{E}_{z}^{n-m-1}-\ddot{E}_{z}^{n-m}\right)\left(\xi^{m}+\xi^{m+1}\right)\right]
\end{aligned}
$$

$$
\sigma^{m}=\int_{m \Delta t}^{(m+1) \Delta t} \sigma(\tau) d \tau=\frac{\varepsilon_{0}}{v}[1-\exp (-v \Delta t)] \exp (-m v \Delta t)
$$

$$
\xi^{m}=\int_{m \Delta t}^{(m+1) \Delta t}(\tau-m \Delta t) \sigma(\tau) d \tau=\frac{\varepsilon_{0}}{v^{2} \Delta t}[1-(1+v \Delta t) \exp (-v \Delta t)] \exp (-m v \Delta t)
$$

$\psi_{z}^{n}=\sum_{m=0}^{n-1}\left[E_{z}^{n-m}\left(\sigma^{m}+\sigma^{m+1}\right)+\left(E_{z}^{n-m-1}-E_{z}^{n-m}\right)\left(\xi^{m}+\xi^{m+1}\right)\right]$

So Eq. 11 can be written as

$\left.J_{z}^{n+1}+J_{z}^{n}=(7) \sigma^{0}-\xi^{0}\right) \ddot{E}_{z}^{n+1}+\xi^{0} \ddot{E}_{z}^{n}+\psi_{z}^{n}$

From Eq. 12, Eq. 13 and Eq. 14

$\psi_{z}^{n}=\left(\sigma^{0}+\sigma^{1}-\xi^{0}-\xi^{1}\right) \ddot{E}_{z}^{n}+\left(\xi^{0}+\xi^{1}\right) \ddot{E}_{z}^{n-1}+\exp (-v \Delta t) \psi_{z}^{n-1}$

In Eq. $7, c f=1-\varepsilon_{1} \sin ^{2} \theta$, and introduce time-varied value $\omega_{p}(t)$ into $3^{\text {rd }}$ equation in Eq. 7 ,

$$
\frac{\partial J_{z}}{\partial t}+v J_{z}=\varepsilon_{0} \omega_{p}^{2}(t) E_{z}
$$

Take difference discrete for $2^{\text {nd }}$ equation in Eq. 10 


$$
\begin{aligned}
(\nabla \times H)_{z}^{n+1 / 2} & =\frac{H_{y}^{n+1 / 2}\left(i+\frac{1}{2}\right)-H_{y}^{n+1 / 2}\left(i-\frac{1}{2}\right)}{\Delta x} \\
& =c f \cdot \varepsilon_{0} \frac{E_{x}^{n+1}\left(i+\frac{1}{2}, j, k\right)-E_{x}^{n}\left(i+\frac{1}{2}, j, k\right)}{\Delta t}+\frac{J_{x}^{n+1}\left(i+\frac{1}{2}\right)+J_{x}^{n}\left(i+\frac{1}{2}\right)}{2}
\end{aligned}
$$

Take Eq. 9 and Eq. 15 into Eq. 18, the electric field intensity can be written as

$$
\begin{aligned}
E_{z}^{n+1}(i) & =\frac{1}{1+\frac{\Delta t}{2 c f \cdot \varepsilon_{0}}\left(\sigma^{0}-\xi^{0}\right) \cdot\left(\omega_{p}^{n+1}\right)^{2}}\left\{\left(1-\frac{\Delta t}{2 c f \cdot \varepsilon_{0}} \xi^{0} \cdot\left(\omega_{p}^{n}\right)^{2}\right) E_{z}^{n}(i)\right. \\
& \left.+\frac{\Delta t}{c f \cdot \varepsilon_{0}}(\nabla \times H)_{z}^{n+1 / 2}-\frac{\Delta t}{2 c f \cdot \varepsilon_{0}} \psi_{z}^{n}(i)\right\}
\end{aligned}
$$

The magnetic field intensity can be derived similarly.

\section{NUMERICAL Analysis AND Validation}

Example: Plasma thickness $\mathrm{d}=5 \mathrm{~cm}$, collision frequency $\mathrm{v}=1 \mathrm{GHz}$, electron density $\mathrm{Ne}=N e_{\text {Refer }} \cdot\left[1+A \cdot \sin \left(2 \pi f_{D} \cdot t\right)\right]$, and $N e_{\text {Refer }}=1 e 18 \mathrm{~m}^{-3}, A=0.15, f_{D}=100 \mathrm{MHz}$. The frequency of incident $\mathrm{EM}$ wave is $10 \mathrm{GHz}$, and incident angle is $0^{\circ}, 30^{\circ}$ and $60^{\circ}$. The calculated results are shown in Fig. 2, and Fig. 2(a) is normalized transmission coefficient and Fig. 2(b) is power transmission coefficient.

It can be seen from Fig. 2 that, when electron density of plasma is periodic varied, there are some other frequency in spectrum in addition to the original frequency, and the spectrum of transmission wave can be written as

$$
\begin{aligned}
\mathcal{F}(f t)= & A_{0} \mathcal{F}(f r)+ \\
& \mathcal{F}(f r) *\left[A_{1} \cdot \mathcal{F}\left(f_{D}\right)+A_{2} \cdot \mathcal{F}\left(2 f_{D}\right)+A_{3} \cdot \mathcal{F}\left(3 f_{D}\right)+\ldots\right]
\end{aligned}
$$

$\mathcal{F}(f t)$ is spectrum of transmission wave, $\mathcal{F}(f r)$ is spectrum of incident wave, $\mathcal{F}\left(n \cdot f_{D}\right)$ is spectrum of sinusoidal wave with $n \cdot f_{D}$ frequency, and the coefficient $A_{0}, A_{1}, A_{2}, A_{3} \ldots$ are smaller than 1 and decrease progressively. The spectrum of transmission wave is similar as the sum of several modulation of incident wave.

We can also conclude from Fig. 2(a) that when the incident angle is large, the transmission distance of EM wave is plasma is large, the attenuation is large and the amplitude of transmission wave is small. It can be seen from Fig. 2(b) that the sub-frequencies in transmission wave are independent to the incident angle, the sub-frequencies is only related to electron density and the gap between subfrequencies is equal to variation frequency of electron density.

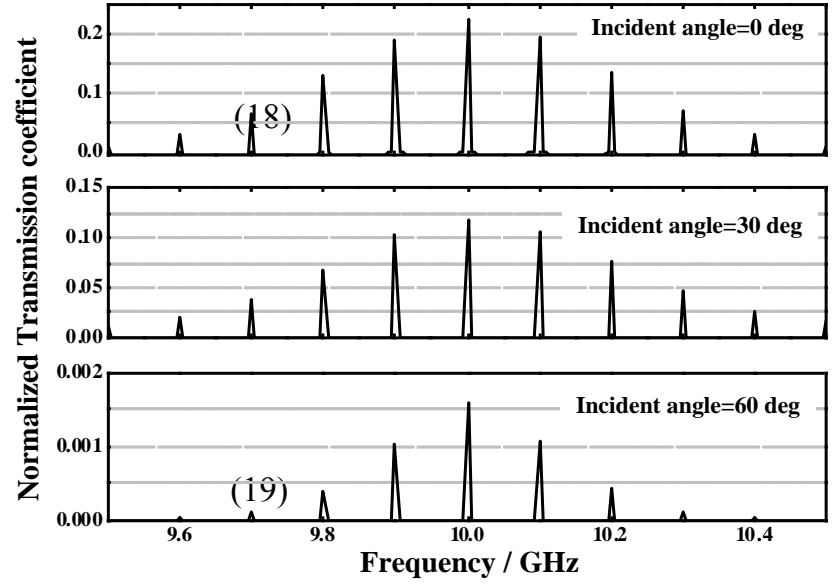

(a)

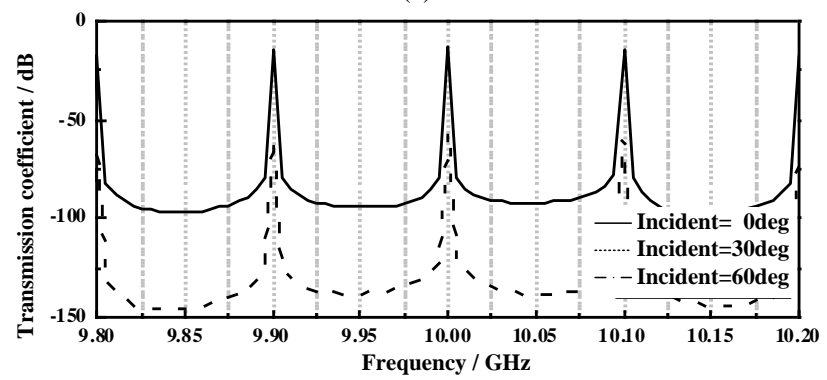

(b)

Figure 2. Transmission spectrum of EM in plasma under different incident angle.

\section{CONCLUSION}

Based on PLJERC FDTD method, one-dimensional FDTD equation for obliquely incident EM wave in timevaried plasma is derived. This method is used to calculate the transmission response of EM wave in plasma with variation of electron density, and good comparison is achieved between numerical results and measurement results.

The influence of EM wave in plasma under different incident angle is analyzed using this method, and it can be concluded that for slow variation plasma, the frequency shift and frequency broaden of transmission wave is large under large incident angle. For periodic variation plasma, subfrequencies in transmission wave are independent to the incident angle, the sub-frequencies is only related to electron density and the gap between sub-frequencies is equal to variation frequency of electron density.

\section{ACKNOWLEDGMENTS}

The author would like to thank the sponsor of National Basic Research Program of China (973 Program, No. 2014CB340205) and National natural Science Foundation of China (NSFC, No. 61431010)

\section{REFERENCES}

[1] J.H. Lee, D.K. Kalluri, C.N. Gary, 2000 International Journal of Infrared and Millimeter Waves 211223 
[2] S.B. Liu, J.J. Mo, N.C. Yuan, 2002 Chinese Journal of Radio Science 17 524(in Chinese)

[3] Mounior Laroussi, J. Reece Roth, 1993 IEEE Trans. Plasma Sci. 21 366

[4] C.W. Scott, K. Panagiotis, 2005 IEEE Trans. Antennas Propag. 53 1721

[5] Y.N. Jiang, D.B. Ge, 2008 Acta Phys. Sin. 57 6283(in Chinese)

[6] L.X. Yang, Y.T. Xie, W. Kong, P.P. Yu, G. Wang, 2010 Acta Phys. Sin. 596089

[7] S.B. Liu, N.C. Yuan, J.J. Mo, 2003 IEEE Microwave and Wireless Components Letters 13187

[8] Z.S. Sacks, D.M. Kingsland, D.M. Lee, J.F. Lee, 1995 IEEE Trans. Antennas Propagat. 431460

[9] S.D. Gedney, 1996 IEEE Trans. Antennas Propagat. 441630 